\title{
ESTUDIO ALTMÉTRICO SOBRE EL ANÁLISIS DE LA PRODUCCIÓN CIENTÍFICA EN RELACIÓN CON LA COVID-19 DESDE LA RED SOCIAL ACADÉMICA RESEARCHGATE (2019-2021)
}

\section{ALTMETRIC STUDY ABOUT THE ANALYSIS OF THE SCIENTIFIC PRODUCTION IN CONNECTION WITH COVID-19 FROM THE ACADEMIC SOCIAL NETWORK RESEARCHGATE (2019-2021)}

\author{
Lisandra María Sosa Valdés ${ }^{1}$, Riselis Martínez Prince ${ }^{2 \star}$, Idania Licea Jiménez ${ }^{3}$, \\ Manuel Licea Puig ${ }^{4}$ \\ 1,2,3 - Universidad de La Habana, La Habana, Cuba. \\ 4 - Instituto Nacional de Endocrinología, La Habana, Cuba.
}

1. Email: lisandram22@nauta.cu ORCID: https://orcid.org/0000-0001-7233-5625

2. Email: riselis.martinez@fcom.uh.cu ORCID: https://orcid.org/0000-0001-8531-5527

3. Email: idania@fcom.uh.cu ORCID: https://orcid.org/0000-0002-1433-3792

4. Email: licea@infomed.sld.cu ORCID: https://orcid.org/0000-0002-9660-5201

Recibido: 11/12/2020 Aceptado: 23/06/2021

Para Citar: Sosa Valdés, L. M., Martínez Prince, R., Licea Jiménez, I., \& Licea Puig, M. (2021). Estudio altmétrico sobre el análisis de la producción científica en relación con la COVID-19 desde la red social académica ResearchGate (2019-2021). Revista Publicando, 8(32), 3-11. https://doi.org/10.51528/rp.vol8.id2199

\begin{abstract}
Resumen:
La investigación presenta el análisis de la producción científica sobre la COVID-19, mediante indicadores altmétricos, en la red social académica ResearchGate durante el período 2019-2021. Se exponen fundamentos teóricos-conceptuales sobre la evaluación de la ciencia, redes sociales académicas y altmetrics. Se caracteriza la red social académica ResearchGate como fuente de estudio. Se propone un estudio descriptivo, no experimental y de corte transversal. La investigación sobre la COVID-19 es un tema que genera gran interés a nivel mundial puesto que se está ante una pandemia de la que se conoce poco y aún no existe un medio efectivo para su control, de ahí que la producción científica sobre la enfermedad continúe creciendo y con ello la necesidad de visualizar y poner a disposición de todos los resultados de investigación, utilizando los medios de comunicación social de la web 2.0. Evaluar la producción científica sobre la COVID-19 en una red social académica, permitirá conocer el impacto y visibilidad académica y social que ha tenido el tema en la comunidad científica y sociedad desde etapas tempranas de la investigación.
\end{abstract}

Palabras clave: producción científica, COVID-19, indicadores altmétricos, ResearchGate, evaluación de la ciencia.

\begin{abstract}
:
The research presents the analysis of the scientific production on COVID-19, using altmetrics indicators, in the academic social network ResearchGate during the period 2019-2021. Theoretical-conceptual foundations on the evaluation of science, academic social networks and altmetrics are exposed. The academic social network ResearchGate is characterized as a source of study. A descriptive, non-experimental and cross-sectional study is proposed. Research on COVID-19 is a topic that generates great interest worldwide since we are facing a pandemic of which little is known and there is still no effective means for its control, hence the scientific production on the disease continues growing and with it the need to visualize and make available to all research results, using the social media of web 2.0. Evaluating the scientific production on COVID-19 in an academic social network will allow to know the impact and academic and social visibility that the subject has had in the scientific community and society from the early stages of the research.
\end{abstract}

Keywords: scientific production, COVID-19, altmetrics indicators, ResearchGate, science evaluation. 


\section{INTRODUCCION}

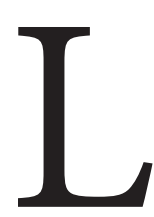

a evaluación de la actividad científica es un aspecto esencial para el desarrollo económico, político y social de cualquier sociedad, toda vez que proporciona las herramientas para discernir entre lo que tiene o no calidad en un contexto donde se fomenta la investigación, cada día se publican nuevos estudios $\mathrm{y}$, asimismo, nuevas innovaciones.

La creciente oleada investigativa que surgió a partir de la segunda guerra mundial ha puesto en el punto de mira la actividad evaluativa científica y ha supuesto nuevos retos para autores $\mathrm{e}$ instituciones. Lo nuevos cambios se insertan en un ambiente cada vez más globalizado, en donde la ciencia, tecnología e innovación ocupan un lugar cimero y son puntales dentro del crecimiento económico y social de un país.

Bajo esta mirada, el hecho de disponer de información que refleje cuantitativamente $y$ cualitativamente los distintos aspectos de las actividades de la $\mathrm{I}+\mathrm{D}$ reviste una importancia vital a la hora de asignar recursos, determinar las políticas de investigación y tecnología y evaluar el impacto de su ejecución (Solís, Milanés y Navarrete, 2010).

En tal sentido, Arencibia y Moya (2008) plantean que

Los indicadores de ciencia y técnica, como constructos sociales, miden aquellas acciones sistemáticas relacionada con la generación, difusión, transmisión y aplicación de conocimientos científicos y tecnológicos. Asimismo, los indicadores bibliométricos constituyen una de las herramientas más utilizadas para la medición del producto de la investigación científica, porque la documentación (independientemente del tipo de soporte) es el vehículo más prolífero y exitoso para la transferencia de conocimiento científico conjuntamente con su transferencia oral por medio de conferencias y comunicaciones personales ( $\mathrm{p}$. 3).

Partiendo de la anterior se concluye que los indicadores son las herramientas que permiten evaluar la actividad científica cuyos resultados se concreta en publicaciones documentales, mediante las cuales se socializa y comunica el conocimiento y a la vez son susceptibles de ser medidas. Los indicadores bibliométricos, por ser los que están vinculados directamente al documento, son los más utilizados.

Siguiendo esta línea los anteriores autores expresan que la producción científica de un país o institución es el conjunto de sus trabajos publicados, en tanto resultados de un proceso de investigación, y los indicadores bibliométricos las medidas que proveen información sobre esos resultados.

El sistema por excelencia para evaluar la actividad científica y con ello la calidad de las publicaciones, como requisito fundamental para formar parte de ese reducido grupo de investigaciones que pudiesen ser financiadas $y$ puestas en marcha, se basa, esencialmente, en el recuento de las citas que ha generado la publicación, dígase revista. De ahí que el Factor de Impacto (FI) fuese el indicador estrella para medir la calidad de la investigación, a partir del número de veces que ha sido citado.

Según Orduña, Martín y Delgado (2016), la unidad de análisis de todos los recuentos bibliométricos ha sido la revista. Los Rankings de 
revistas (...) ordenados por su Impact Factor (IF) y publicados anualmente por el antiguo Institute for Scientific Information (ISI) con ediciones separadas para las ciencias naturales y sociales, proporcionaban los índices indiscutibles para evaluar el rendimiento de toda actividad científica. Un autor o una institución valían lo que valiera la propia revista donde se publicara su trabajo.

Nótese que estos autores se refieren al IF como medida indiscutible para evaluar calidad científica, pero no del documento científico en sí (artículo) sino de la revista donde se publicara dicho artículo, de manera tal que este último recibe el FI de la revista. Durante años el FI, de conjunto con el índice $\mathrm{H}_{2}$ (surgido posteriormente) fueron los indicadores más usados para evaluar el rendimiento científico. Sin embargo, ese mismo tiempo ha servido para conocer en profundidad las potenciales, pero igualmente limitaciones del FI. Por una parte, las Ciencias Sociales son el campo de estudio más afectado con la utilización del FI si se tiene en cuenta que la mayoría de las revistas publicadas en el Index Citation Reports pertenecen a las ciencias naturales y exactas (matemáticas, físicas y otras), puesto que son los campos de estudios donde se hace más palpable la innovación científica-tecnológica, sus resultados y puesta en marcha. Por otro lado, el FI se enfoca básicamente en la revista y no el contenido de esta, que son los artículos y sus autores.

Lo anterior aparejado al desarrollo de las TIC trajo como consecuencia el surgimiento de nuevos indicadores bibliométricos basados en un cambio en la unidad de análisis y una explosión de nuevas unidades de medida. Estos nuevos indicadores bibliométricos basados no en la revista y si en el artículo y el autor se denominan altmetrics (author-level metrics y article level metrics). Esta medición debe enfocarse, desde dos niveles diferentes (Orduña, Martín y Delgado, 2016, p. 488):

- El impacto académico de un artículo no debe ceñirse exclusivamente a las citas recibidas desde otros artículos indizados en ciertas bases de datos bibliográficas.

- El impacto académico no debe restringirse exclusivamente al artículo, existiendo otros documentos (cuyo grado de uso es susceptible de ser cuantificado) de gran valor para la comprensión del impacto académico (presentaciones, software, datos, etc.).

Las altmétricas son un conjunto de indicadores (el número de comparticiones, de redifusiones de un artículo o los comentarios que ha generado, entre otros) que miden la presencia y el eco que ha tenido una publicación en las redes sociales y académicas (Abadal, 2018).

Este mismo autor plantea que las altmétricas, por otra parte, valoran el eco social de cada artículo y no de una revista en su conjunto. Se denominaron altmétricas a este conjunto de indicadores que ofrecen una visión complementaria a la rigidez de las citas. Sus datos son el máximo interés para los autores, los lectores y también para los financiadores de la investigación, ya que miden el uso y el eco social de las publicaciones científicas.

Partiendo de ello, se concluye que Altmetrics son un conjunto de indicadores alternativos (a la bibliometría actual, al análisis de citas y al FI) que permiten medir el impacto social de una investigación a partir de las informaciones expuestas en redes sociales académicas y 
plataformas sociales e investigativas.

El desarrollo de las TIC no solo dio origen a las altmetrics sino también a nuevas formas de comunicar la investigación utilizando las potenciales y herramientas que brinda la Web 2.0, cuya teoría defiende la interacción entre usuarios y el poder compartir contenidos. Tal es el caso de las redes sociales académicas.

Se habla de los sitios o redes sociales como los servicios basados en web que están atrayendo cada vez más a académicos e investigadores con una triple finalidad: construir un perfil público o semipúblico dentro de un sistema acotado, formalizar una lista de usuarios con los que establecer una conexión y ver y cruzar la propia lista de contactos hechas por otros usuarios dentro del sistema (Hernández y Fernández, 2018).

En definitiva, el objetivo de las redes sociales es propiciar la comunicación, integración $y$ cooperación, entre sus miembros y compartir información. "Las redes sociales son el mejor exponente del trabajo de la Web 2.0 (...)" (Hernández y Fernández, 2018, p.3). En este sentido, la red social académica ResearchGate es un claro ejemplo de lo anterior.

Fundada en Alemania en el año 2008 (...), el sistema se basa fundamentalmente en la capacidad para depositar y almacenar cualquier documento académico por parte de los autores (desde un artículo publicado en una revista de impacto hasta patentes, comunicaciones a congresos, materiales de un curso, una presentación o datasets) y en la inmediata obtención de estadísticas de su uso personalizadas (quien visita, descarga o cita un documento o a sus autores). (Orduña, Martín y Delgado, 2016, p.304)

Es una red social para académicos que permite visibilizar y socializar publicaciones científicas que aborden diversas temáticas y a través de la cual se puede medir el impacto social, utilizando, entre otros, indicadores altmétricos.

La investigación en el área de la salud es un campo muy prolífero por las aplicaciones y resultados que proporciona, es por ello que los medios sociales de comunicación científica actuales se hacen eco de nuevas investigaciones de este tipo. En estos últimos tiempos, la actividad investigativa que más ha crecido y desarrollado gira en torno a la pandemia de la COVID-19 como resultado de la emergencia sanitaria $y$ epidemiológica que se vive en el mundo entero. Se publica rápidamente sobre el tema y los autores ponen a disposición de la comunidad (científica o no) los resultados de investigación mediante múltiples plataformas informativas entre las que destacan las redes sociales académicas por la interacción y repercusión social que ofrecen, en este caso ResearchGate posee una amplia producción científica en relación a este fenómeno, que crece cada día.

El virus descubierto es un coronavirus novedoso mutado (género B), que es nombrado SARS-CoV-2 por la Organización Mundial de la Salud y por el Comité Internacional para la Taxonomía del Virus. Como consecuencia de las mutaciones antigenéticas del coronavirus, este se considera un nuevo virus para los humanos y la población en general carece de inmunidad contra la nueva cepa (Ortiz, 2020, p.3).

La enfermedad por COVID-19 o novel coronavirus, es una pandemia global de enfermedad respiratoria aguda causada por este virus, que filogenéticamente está estrechamente 
relacionado con el SARS-CoV. Comenzó en diciembre de 2019 en Wuhan, provincia de Hubei en China y fue declara pandemia global el 11 de marzo de 2020.

Zayas, Madero, Rodríguez y Alfonso (2020) afirman que "mientras el brote del coronavirus tipo 2 del síndrome respiratorio agudo grave o SARS-CoV-2 se ha expandido por 185 países, la cooperación científica ha permitido abrir nuevos circuitos para compartir información y ganarle terreno al coronavirus, por lo que una buena práctica internacional ha sido una acelerada reconversión hacia el acceso abierto" (p.4).

La pandemia ha revolucionado todos los aspectos de la vida social, pero también investigativa. Desde esta perspectiva es esencial conocer cuál ha sido el comportamiento sobre la COVID-19 en cuanto a publicación se refiere, teniendo en cuenta que toda información sobre este tema resulta insuficiente en el contexto actual, que requiere de una alta dosis de conocimiento sobre un virus, no tan desconocido, pero del que sí, aún, se buscan respuestas, soluciones y curas. ResearchGate como una red social académica de acceso abierto se ha convertido en una potente plataforma informativa para socializar $y$ comunicar investigaciones asociadas a este tema desde el inicio de la pandemia.

\section{PLANTEAMIENTO DEL PROBLEMA}

Las altmetrics o métricas alternativas como herramientas para medir la ciencia es una temática novedosa y en creciente desarrollo dentro de los estudios métricos. Las investigaciones al respecto están asociadas a la definición de altmetrics como nuevos indicadores para medir el impacto $y$ difusión científico frente a los métodos tradicionales de medición de impacto; asimismo su empleo en las plataformas y medios de comunicación social que ofrece la web 2.0.

A pesar de ello, muy pocos estudios han abordado las potencialidades y utilidad de esta herramienta para medir visibilidad e impacto académico y social sobre una temática determinada en una red social académica, lo que impide su aprovechamiento para conocer desde etapas tempranas de la investigación el impacto y relevancia social que ha tenido el tema en autores y publicaciones y como se ha comportado la productividad científica en ese campo durante un período de tiempo.

\section{PREGUNTA DE INVESTIGACIÓN}

¿Cuál es el comportamiento de la producción científica sobre la COVID-19, a partir de indicadores altmétricos, en la red social académica ResearchGate durante el período 2019-2021?

\section{SISTEMA DE OBJETIVOS}

\section{Objetivo general}

Analizar el comportamiento de la producción científica sobre la COVID-19, a partir de indicadores altmétricos, en la Red Académica ResearchGate durante el período 2019-2021.

\section{Objetivos específicos}

1. Abordar fundamentos teóricosconceptuales sobre la evaluación de la investigación científica, las Altmetrics y las redes sociales académicas.

2. Caracterizar la Red Social Académica ResearchGate como fuente de datos para el estudio. 
3. Construir la batería de indicadores altmétricos a aplicar.

4. Caracterizar el comportamiento cuantitativo y cualitativo de la producción científica sobre la COVID-19 en la Red Académica ResearchGate durante el período 2019-2021, a través de indicadores altmétricos.

\section{MÉTODOS Y TÉCNICAS}

- Análisis documental clásico: permite recopilar la información existente sobre evaluación de la investigación científica, altmetrics, ResearchGate y la enfermedad COVID-19 para el desarrollo del marco teórico-conceptual de la investigación.

- Análisis de Contenido Cualitativo: a fin de, por un lado, explicar el porqué de los resultados métricos obtenidos y, por el otro, identificar, analizar e interpretar el contenido semántico de los conceptos correspondiente a los términos efectivamente utilizados en determinados textos (Magariños, 2010), dígase, por ejemplo, el análisis de la coocurrencia de palabras clave para la identificación de líneas de investigación importantes dentro del área de conocimientos que se analiza.

- Métodos de los Estudios Métricos de la Información: empleados para obtener $y$ cuantificar los resultados obtenidos, y para determinar el comportamiento de la producción científica sobre la COVID-19 a partir de la aplicación de indicadores altmétricos durante un período de estudio.

- Técnicas de Visualización de la Información: utilizadas para la representación y visualización de los resultados a partir del trabajo con software.

\section{JUSTIFICACIÓN}

Los cambios que se han producido en la manera de comunicar la ciencia debido fundamentalmente al surgimiento de internet y las plataformas de comunicación sociales que ofrece la web 2.0 han supuesto, igualmente, cambios en las formas para medir el impacto, relevancia y visibilidad de una publicación. Los métodos tradicionales para medir la ciencia poseen limitaciones en el entorno de la web, caracterizada por la inmediatez, visibilidad y repercusión social que brinda.

Según, Williams y Padula (2015), "Si bien las métricas a nivel de revista tenían sentido en la década de 1950, cuando se asociaba un artículo a una revista impresa; en el mundo digital la asociación con una revista impresa es menos consecuente" (p.7). Sumado a ello, indicadores como el FI y el recuento de citas requieren de un período de observación más amplio para determinar el impacto o no de una publicación.

Desde hace algunos años se habla de altmetrics como una herramienta alternativa para medir visibilidad e impacto en las plataformas que ofrece la web 2.0; útil para analizar las diversas formas de comunicación científica y la medición del impacto no solo académico sino también social de determinada temática en tiempo real.

La COVID-19 es el tema de investigación en el que más se trabaja y publica hoy día, toda vez que se trata de una pandemia que ha paralizado el mundo entero y para la que aún no hay un medio de control disponible. El incremento de la producción científica sobre la enfermedad y el rol 
primordial que están asumiendo los medios de comunicación científica sociales en la web para la difusión y visibilidad de los resultados investigativos, propician el escenario adecuado para medir esa actividad científica utilizando indicadores altmétricos.

Tomando en consideración lo antes expuesto y teniendo en cuenta la importancia de altmetrics como herramienta para la evaluación de la ciencia en el entorno de la web, la investigación propuesta contribuirá al análisis de la visibilidad e impacto académico y social que ha tenido la COVID-19 en una plataforma social académica; así como también el comportamiento de la producción científica sobre la pandemia desde la primera etapa de la investigación y hasta la actualidad (2019-2021).

Por otro lado, no son pocos los autores que afirman que las redes sociales constituyen la primera fuente de información científica de los ciudadanos y tienen un papel primordial como herramienta de comunicación científica, dado por su relevancia social y las múltiples potencialidades y prestaciones de uso que tienen.

En este contexto, las redes sociales científicas son esenciales para el intercambio, difusión y visualización de información científica. Son plataformas desde la cuales se pueden descargar y compartir publicaciones en un ambiente de socialización y creación de impacto; asimismo, sirven para saber el interés social que ha generado un tema, artículo y autor.

Desde esta perspectiva, mediante la investigación se podrá conocer las características y prestaciones de una red social académica, con énfasis en su capacidad para difundir y socializar los resultados de investigación sobre una temática en el menor tiempo posible y con ello la generación de impacto académico y social. Finalmente, el estudio servirá de referente para futuras investigaciones sobre el tema.

\section{TIPO DE INVESTIGACIÓN}

Esta investigación se considera cuantitativa con alcance descriptivo. El aspecto cuantitativo se refleja en los datos estadísticos recogidos por las herramientas empleadas para el análisis altmétrico, a través de indicadores altmétricos y bibliométricos. El aspecto cualitativo se utiliza para explicar los resultados alcanzados luego de la recogida y análisis de los datos estadísticos.

El estudio es descriptivo, no experimental y de corte transversal ya que se determina, durante el período 2019-2021, el comportamiento de la producción científica sobre la COVID-19 en la red social académica ResearchGate utilizando indicadores altmétricos y bibliométricos. Se observa el fenómeno en su contexto natural. Se describe la variable asociada al estudio y su incidencia en dicha red social académica durante el período escogido.

\section{VARIABLES}

\section{Variable teórica: producción científica}

Definición conceptual: conjunto de conocimientos que han sido generados como efecto del proceso de investigación en cualquier rama del saber llevado a cabo por una persona determinada, siendo este el punto de partida para generar nuevos conocimientos en cualquier profesional (Gámez, 2017, p. 24). 
Definición real: forma en que se manifiesta la producción documental y de conocimiento sobre la COVID-19 en la red social académica ResearchGate entre los años 2019-2021.

\section{CONCLUSIONES}

- La pandemia ha revolucionado todos los aspectos de la vida social, pero también investigativa. Desde esta perspectiva es esencial conocer cuál ha sido el comportamiento sobre la COVID-19 en cuanto a publicación se refiere, teniendo en cuenta que toda información sobre este tema resulta insuficiente en el contexto actual, que requiere de una alta dosis de conocimiento sobre un virus, no tan desconocido, pero del que sí, aún, se buscan respuestas, soluciones, curas y se toma decisiones.

- ResearchGate como una red social académica de acceso abierto se ha convertido en una potente plataforma informativa para socializar y comunicar investigaciones asociadas a este tema desde el inicio de la pandemia y evaluar la producción científica sobre la temática en dicha red, contribuirá al conocimiento del impacto y visibilidad académica y social que ha tenido el tema en la comunidad científica y sociedad desde etapas tempranas de esta investigación.

- La investigación aspira a ofrecer una panorámica del comportamiento autoral y documental de la producción científica sobre la COVID-19 en la red social académica ResearchGate, durante el período 2019-2021. Para ello se propone utilizar indicadores altmétricos que permitan evaluar el quehacer científico desde un enfoque social y académico. En este sentido, se espera conocer, entre otros aspectos:

- Los autores con más publicaciones en la plataforma y el tipo de publicación;

- El impacto académico mediante el número de citas por autor y tipo de documento;

- Los documentos más leídos, descargados a texto completo y recomendados por los usuarios registrados en la plataforma;

- El impacto social a través de la interacción de los autores con usuarios y comunidad científica. En este apartado se pretende saber los autores y usuarios seguidos y perfiles más visitados.

.


$\begin{array}{lllllll}\text { R } & \text { E } & \text { V } & \text { I } & \text { S } & \text { T } & \text { A }\end{array}$

Public a n d o

I S S N $133900-9304$

\section{REFERENCIAS}

Abadal, E. (2018). Las altmétricas: aportaciones para la evaluación de publicaciones científicas en ciencias humanas y sociales. Revista PH, (95), 136-147.

Alonso, J., Cordón-García, J., y Maltrás, B. (2016). Altmetrics: medición de la influencia de los medios en el impacto social de la investigación. Cuadernos de Documentación Multimedia, 27(1), 75-101. doi: http://dx.doi.org/10.5209/ rev_CDMU.2016.v27.n1.52870

Arencibia Jorge, R., y Moya Anegón, F. d. (2008). La evaluación de la investigación científica: una aproximación teórica desde la Cienciometría. Acimed, 17(4), 127.

Castro L. (2020). Coronavirus, una historia en desarrollo. Revista Médica de Chile, 148, 143-144.

Gámez Ramírez, M. (2017). Análisis de la Producción científica de los doctores de la Universidad de Guantánamo en el período de 2012-2016. (Trabajo de Diploma), Instituto Superior Minero Metalúrgico de Moa Dr. Antonio Núñez Jiménez, Moa.

Hernández, J., y Fernández, B. (2018). Las Redes sociales científicas y académicas. Impacto en la visibilidad de la producción científica en Salud Ocupacional. Paper presented at the Convención Internacional de Salud, Cuba.

Magariños, J. (2010). Fundamentos para un análisis del discurso vigente en determinada comunidad. Recuperado el 20 de abril de 2021, de http:// www.semiotica-online.com.ar/ 1Sintesisanálisisoinion.html

Ollé, C., y López-Borrull, A. (2017). Redes sociales y altmetrics: nuevos retos para las revistas científicas. En U. B. Edicions (Ed.), Revistas científicas: situación actual y retos del futuro (pp. 197-219). Barcelona.

Orduña-Malea, E., Martín-Martín, A., y DelgadoLópez-Cózar, E. (2016). La bibliometría que viene: Almetrics (Author Level Metics) y las múltiples caras del impacto de un autor. El profesional de la información, 25(3), 485-496. doi: http:// dx.doi.org/10.3145/epi.2016.may.18

Ortiz-Núñez, R. (2020). Análisis métrico de la producción científica sobre COVID-19 en SCOPUS. Revista Cubana de Información en Ciencias de la Salud, 31(3), 1-20.

Solís Cabrera, F. M., Milánes Guisado, Y., y Navarrete Cortés, J. (2010). Evaluación de la investigación científica. El caso de Andalucía. Revista Fuentes, 10, 83-100.

Williams, C. y Padula, D. (2015). The evolution of impact indicators: from bibliometrics to altmetrics. Scholastica, 31.

Zayas, R., Madero, S., Rodríguez, B., y Alfonso, J. (2020). Producción científica sobre la COVID-19 en revistas médicas cubanas a 90 días del inicio de la pandemia. Revista Habanera de Ciencias Médicas, 19(4). 